PROCEEDINGS OF THE AMERICAN MATHEMATICAL SOCIETY

Volume 124, Number 10, October 1996

\title{
NONLINEAR OSCILLATIONS OF SECOND ORDER DIFFERENTIAL EQUATIONS OF EULER TYPE
}

\author{
JITSURO SUGIE AND TADAYUKI HARA
}

(Communicated by Hal L. Smith)

Dedicated to Professor Junji Kato on the occasion of his 60th birthday

\begin{abstract}
We consider the nonlinear equation $t^{2} x^{\prime \prime}+g(x)=0$, where $g(x)$ satisfies $x g(x)>0$ for $x \neq 0$, but is not assumed to be sublinear or superlinear. We discuss whether all nontrivial solutions of the equation are oscillatory or nonoscillatory. Our results can be applied even to the case $\frac{g(x)}{x} \rightarrow \frac{1}{4}$ as $|x| \rightarrow$ $\infty$, which is most difficult.
\end{abstract}

\section{InTRODUCTION AND STATEMENT OF RESULts}

We consider the oscillation problem for the second order nonlinear differential equation

$$
t^{2} x^{\prime \prime}+g(x)=0, \quad t>0,
$$

where $g(x)$ is locally Lipschitz continuous on $\mathbf{R}$ and

$$
x g(x)>0 \quad \text { if } \quad x \neq 0 .
$$

A nontrivial solution of (1.1) is said to be oscillatory if it has arbitrarily large zeros. Otherwise, the solution is said to be nonoscillatory. In the theory of oscillations, the number $\frac{1}{4}$ very often appears as a critical value. The following result is a good illustration of this fact: all nontrivial solutions of Euler's equation

$$
t^{2} x^{\prime \prime}+\lambda x=0
$$

are oscillatory if and only if $\lambda>\frac{1}{4}$. Other examples are found in [3], [6] and the references cited therein.

Because of Sturm's separation theorem, the solutions of second order linear differential equations are either all oscillatory or all nonoscillatory, but cannot be both. Thus, we can classify second order linear differential equations into the two types. However, the oscillation problem for (1.1) is not so easy, because $g(x)$ is nonlinear.

Judging from the oscillation result for Euler's equation (1.3), we see that all nontrivial solutions of (1.1) have a tendency to be oscillatory as $g(x)$ grows larger

Received by the editors April 6, 1995.

1991 Mathematics Subject Classification. Primary 34C10, 34C15; Secondary 34A12, 70 K05.

Key words and phrases. Oscillation, nonlinear differential equations, Liénard system, global phase portrait.

The first author was supported in part by Grant-in-Aid for Scientific Research 06804008.

(C)1996 American Mathematical Society 
in some sense; and we must consider the case

$$
\frac{g(x)}{x} \rightarrow \frac{1}{4} \quad \text { as } \quad|x| \rightarrow \infty
$$

to solve completely the oscillation problem for (1.1).

The purpose of this paper is to give our answer to this delicate problem. Our main results are stated in the following:

Theorem 1.1. Let $\lambda>0$. Then all nontrivial solutions of (1.1) are oscillatory if

$$
\frac{g(x)}{x} \geq \frac{1}{4}+\frac{\lambda}{\log |x|}
$$

for $|x|>R$ with a sufficienlty large $R>0$.

Theorem 1.2. Suppose that there exists a $\lambda$ with $0<\lambda<\frac{1}{4}$ such that

$$
\frac{g(x)}{x} \leq \frac{1}{4}+\left(\frac{\lambda}{\log |x|}\right)^{2}
$$

for $x>R$ or $x<-R$ with a sufficiently large $R>0$. Then all nontrivial solutions of (1.1) are nonoscillatory.

Remark 1.1. We note that condition (1.4) is satisfied in either case

or

$$
g(x)=\frac{1}{4} x+\frac{\lambda x}{\log |x|} \quad \text { with } \quad \lambda>0
$$

$$
g(x)=\frac{1}{4} x+\left(\frac{\lambda}{\log |x|}\right)^{2} x \quad \text { with } \quad 0<\lambda<\frac{1}{4}
$$

for $|x|$ sufficiently large.

\section{Global existence of SOlutions}

In this section we will show that each solution of (1.1) exists in the future. To see this, we consider the following system

$$
\begin{aligned}
& x^{\prime}=y \\
& y^{\prime}=-\frac{g(x)}{t^{2}}
\end{aligned}
$$

equivalent to (1.1).

Proposition 2.1. Assume (1.2). Then every solution of (2.1) exists in the future.

To prove Proposition 2.1, we supply a continuation result for the general system

$$
\begin{aligned}
x^{\prime} & =F_{1}(t, x, y) \\
y^{\prime} & =F_{2}(t, x, y)
\end{aligned}
$$

in which $F_{1}:[0, \infty) \times \mathbf{R}^{m} \times \mathbf{R}^{n} \rightarrow \mathbf{R}^{m}$ and $F_{2}:[0, \infty) \times \mathbf{R}^{m} \times \mathbf{R}^{n} \rightarrow \mathbf{R}^{n}$ with both functions being continuous.

A continuous function $V:[0, \infty) \times \mathbf{R}^{m} \times \mathbf{R}^{n} \rightarrow \mathbf{R}$ is called a Liapunov function for $(2.2)$ if $V(t, x, y)$ is locally Lipschitz in $(x, y)$. We define

$$
\dot{V}_{(2.2)}(t, x, y)=\limsup _{h \rightarrow 0+} \frac{1}{h}\left\{V\left(t+h, x+h F_{1}(t, x, y), y+h F_{2}(t, x, y)\right)-V(t, x, y)\right\} .
$$


We also say that a continuous scalar function $\phi:[0, \infty) \times \mathbf{R} \rightarrow \mathbf{R}$ is of the class $\mathcal{G}$ if, for any $t_{0} \geq 0, u_{0} \in \mathbf{R}$, the maximal solution $u\left(t, t_{0}, u_{0}\right)$ of the equation

$$
u^{\prime}=\phi(t, u)
$$

exists in the future.

In [2], [4], [5], the continuation problem is discussed by means of two Liapunov functions for $(2.2)$ :

Proposition 2.2. Let $V:[0, \infty) \times \mathbf{R}^{m} \times \mathbf{R}^{n} \rightarrow \mathbf{R}$ be a Liapunov function with the property that

$$
\begin{aligned}
& V(t, x, y) \rightarrow \infty \quad \text { as } \quad\|y\| \rightarrow \infty \quad \text { uniformly in } x \in \mathbf{R}^{m} \\
& \text { for each fixed } t ;
\end{aligned}
$$

Suppose that for each $K>0$ and $T>0$ there exists a Liapunov function $W$ : $[0, T] \times \mathbf{R}^{m} \times S_{K}^{n} \rightarrow \mathbf{R}, S_{K}^{n}=\left\{y \in \mathbf{R}^{n}:\|y\| \leq K\right\}$ which satisfies

$$
\begin{aligned}
& W(t, x, y) \rightarrow \infty \quad \text { as } \quad\|x\| \rightarrow \infty \quad \text { uniformly in } y \in S_{K}^{n} \\
& \text { for each fixed } t ;
\end{aligned}
$$$$
\dot{W}_{(2.2)}(t, x, y) \leq \psi(t, W(t, x, y)) \quad \text { for some } \psi \in \mathcal{G} .
$$

Then every solution of (2.2) exists in the future.

Using Proposition 2.2, we can prove Proposition 2.1.

Proof of Proposition 2.1. Let $G(x)=\int_{0}^{x} g(\xi) d \xi$. Then we have

$$
G(x) \geq 0 \quad \text { for } \quad x \in \mathbf{R}
$$

by (1.2). Hence, condition (2.3) is satisfied when

$$
V(t, x, y)=\frac{1}{2} y^{2}+\frac{G(x)}{t^{2}} .
$$

Define another Liapunov function

$$
W(t, x, y)=|x|
$$

Clearly, this satisfies condition (2.5). We obtain

$$
\dot{V}_{(2.1)}(t, x, y)=-\frac{2}{t^{3}} G(x) \leq 0
$$

and

$$
\dot{W}_{(2.1)}(t, x, y) \leq|y| \leq K \quad \text { on } \quad S_{K}^{1} .
$$

Since $\phi(t, u)=0$ and $\psi(t, u)=K$ belong to $\mathcal{G}$, conditions (2.4) and (2.6) are satisfied. Thus, by Proposition 2.2 all solutions of (2.1) are continuable in the future.

Remark 2.1. Proposition 2.1 shows that each solution of (1.1) and its derivative exist in the future.

Remark 2.2. The conclusion of Proposition 2.1 is still true if (1.2) is replaced by the more general condition

$$
G(x) \geq-d \quad \text { for } \quad x \in \mathbf{R},
$$

where $d$ is an arbitrary constant. 


\section{Some LEMmas}

The change of variable $t=e^{s}$ reduces (1.1) to the equation

$$
\ddot{x}-\dot{x}+g(x)=0, \quad s \in \mathbf{R},
$$

where $=\frac{d}{d s}$. This equation is equivalent to the system

$$
\begin{aligned}
& \dot{x}=y+x \\
& \dot{y}=-g(x)
\end{aligned}
$$

which is of Liénard type. Note that every solution of (3.1) exists in the future by Proposition 2.1.

In this section we give some results on the asymptotic behavior of trajectories of (3.1). We write $\gamma^{+}(P)$ for the positive semitrajectory of (3.1) starting at a point $P \in \mathbf{R}^{2}$.

Lemma 3.1. For each point $P=(p,-p)$ with $p>0$, the positive semitrajectory $\gamma^{+}(P)$ crosses the negative $y$-axis.

Proof. Suppose that there exists a point $P=(p,-p)$ with $p>0$ such that $\gamma^{+}(P)$ does not intersect the negative $y$-axis. Let $(x(s), y(s))$ be a solution of (3.1) defined on an interval $\left[s_{0}, \infty\right)$ with $\left(x\left(s_{0}\right), y\left(s_{0}\right)\right)=P$. Then the solution $(x(s), y(s))$ corresponds to $\gamma^{+}(P)$. Taking into account the vector field of (3.1), we see that

$$
0<x(s) \leq x\left(s_{0}\right) \quad \text { for } \quad s \geq s_{0}
$$

and

$$
y(s) \rightarrow-\infty \quad \text { as } \quad s \rightarrow \infty .
$$

Hence, it follows from the first equation of (3.1) that

$$
\dot{x}(s) \rightarrow-\infty \quad \text { as } \quad s \rightarrow \infty,
$$

and therefore, there exists an $s_{1}>s_{0}$ such that

$$
\dot{x}(s) \leq-1 \quad \text { for } \quad s \geq s_{1} .
$$

Integration of the above leads to

$$
-x\left(s_{1}\right)<x(s)-x\left(s_{1}\right) \leq s_{1}-s \rightarrow-\infty \quad \text { as } \quad s \rightarrow-\infty .
$$

This is a contradiction and completes the proof.

Similarly, turning our attention to the left half-plane, we have the following result.

Lemma 3.2. For each point $P=(-p, p)$ with $p>0$, the positive semitrajectory $\gamma^{+}(P)$ crosses the positive $y$-axis.

We introduce here a new important concept which is useful in the theory of oscillations. We say that system $(3.1)$ has property $\left(X^{+}\right)$in the right half-plane (resp., left half-plane) if, for every point $P$ in the region $\{(x, y): x \geq 0$ and $y>-x\}$ (resp., $\{(x, y): x \leq 0$ and $y<-x\})$, the positive semitrajectory $\gamma^{+}(P)$ crosses the curve $y=-x$. In [1] the authors went into details about property $\left(X^{+}\right)$ and gave some necessary conditions and some sufficient conditions for property $\left(X^{+}\right)$. We state below special cases of those results. Let

$$
G(\infty)=\int_{0}^{\infty} g(\xi) d \xi \quad \text { and } \quad G(-\infty)=\int_{0}^{-\infty} g(\xi) d \xi
$$


Lemma 3.3 ([1, Theorem 4.1]). Assume $G(\infty)<\infty$ (resp., $G(-\infty)<\infty)$. Then system (3.1) fails to have property $\left(X^{+}\right)$in the right half-plane (resp., left halfplane).

Lemma 3.4 ([1, Theorem 5.4]). Assume $G(\infty)=\infty$ (resp., $G(-\infty)=\infty)$. Then system (3.1) fails to have property $\left(X^{+}\right)$in the right half-plane (resp., left halfplane) if

$$
|x| \geq 2 \sqrt{2 G(x)}-h(\sqrt{2 G(x)}) \quad \text { for }|x| \text { sufficiently large, }
$$

where $h(r)$ is a continunous function on $[0, \infty)$ such that for $r$ sufficiently large

$$
\begin{gathered}
\frac{h(r)}{r} \text { is nonincreasing and nonnegative; } \\
r\left(\int_{r}^{\infty} \frac{h(\xi)}{\xi^{2}} d \xi\right)^{2} \leq \frac{1}{4} h(r) .
\end{gathered}
$$

Lemma 3.5 ([1, Theorem 5.2]). Assume $G(\infty)=\infty$ (resp., $G(-\infty)=\infty)$. Then system (3.1) has property $\left(X^{+}\right)$in the right half-plane (resp., left half-plane) if

$$
|x| \leq 2 \sqrt{2 G(x)}-h(\sqrt{2 G(x)}) \quad \text { for }|x| \text { sufficiently large, }
$$

where $h(r)$ is a continunous function on $[0, \infty)$ with

$$
\begin{aligned}
& \frac{h(r)}{r} \text { is nonincreasing, nonnegative } \\
& \text { and is not greater than } 2 \text { for } r \text { sufficiently large; }
\end{aligned}
$$

$$
\int^{\infty} \frac{h(r)}{r^{2}} d r=\infty
$$

\section{Proof of the theorems}

Proof of Theorem 1.1. As shown in Section 2, each solution of (1.1) exists in the future. Suppose that system (3.1) which is equivalent to (1.1) has property $\left(X^{+}\right)$ in the right and left half-plane. Then it follows from Lemmas 3.1 and 3.2 that every solution of (3.1) keeps on rotating around the origin except the zero solution. Hence, all nontrivial solutions of (1.1) are oscillatory. Thus, to prove Theorem 1.1, it is enough to show that system (3.1) has property $\left(X^{+}\right)$in the right and left half-plane. We will demonstrate this fact by means of Lemma 3.5. Note that (1.5) implies $G( \pm \infty)=\infty$.

Let $0<\nu<\lambda$ and

$$
h(r)=\frac{\nu r}{\log r}
$$

for $r$ sufficienlty large. Then it is clear that conditions (3.6) and (3.7) are satisfied. We next define continuous functions $k(x), K(x)$ and $L(x)$ on $\mathbf{R}$ by

$$
k(x)=\frac{\lambda x}{\log |x|}, \quad K(x)=\int_{0}^{x} k(\xi) d \xi \quad \text { and } \quad L(x)=\frac{\lambda x^{2}}{2 \log |x|}
$$

for $|x|$ sufficiently large, respectively. Then we have

$$
K(x) \geq L(x)-M \quad \text { for some } M>0
$$


and by (1.5)

$$
G(x) \geq \frac{1}{8} x^{2}+K(x)-N \quad \text { for some } N>0 .
$$

Since $x K(x)$ is increasing for $|x|$ sufficiently large, we get

$$
\begin{aligned}
K\left(2 u-\frac{\nu u}{\log |u|}\right)-\frac{\nu u^{2}}{2 \log |u|} & \geq K(u)-\frac{\nu u^{2}}{2 \log |u|} \\
& \geq L(u)-M-\frac{\nu u^{2}}{2 \log |u|} \\
& \geq \frac{(\lambda-\nu) u^{2}}{2 \log |u|}-M
\end{aligned}
$$

which tends to $\infty$ as $|u| \rightarrow \infty$. Hence, for $|u|$ sufficiently large

$$
\begin{aligned}
\frac{1}{2} u^{2} & \leq \frac{1}{2} u^{2}+K\left(2 u-\frac{\nu u}{\log |u|}\right)-\frac{\nu u^{2}}{2 \log |u|}-N+\frac{\nu^{2} u^{2}}{8(\log |u|)^{2}} \\
& =\frac{1}{8}\left(2 u-\frac{\nu u}{\log |u|}\right)^{2}+K\left(2 u-\frac{\nu u}{\log |u|}\right)-N \\
& \leq G\left(2 u-\frac{\nu u}{\log |u|}\right),
\end{aligned}
$$

namely,

$$
\frac{1}{2} u^{2} \leq\left\{\begin{array}{lll}
G(2 u-h(u)) & \text { if } & u>0 \\
G(2 u+h(-u)) & \text { if } & u<0
\end{array}\right.
$$

Letting

$$
u= \begin{cases}\sqrt{2 G(x)} & \text { if } \quad x>0 \\ -\sqrt{2 G(x)} & \text { if } \quad x<0\end{cases}
$$

we have

$$
|x| \leq 2 \sqrt{2 G(x)}-h(\sqrt{2 G(x)})
$$

for $|x|$ sufficiently large, that is, condition (3.5) is also satisfied. Thus, by Lemma 3.5 system $(3.1)$ has property $\left(X^{+}\right)$in the right and left half-plane. The proof is complete.

To prove Theorem 1.2, we need Lemmas 4.1 and 4.2 below.

Lemma 4.1. Every solution of (3.1) is unbounded except the zero solution.

Proof. By way of contradiction, we suppose that there exists a bounded solution $(x(s), y(s))$ of (3.1) initiating at $s=s_{0}>0$, that is,

$$
|x(s)|+|y(s)| \leq B \quad \text { for } \quad s \geq s_{0}
$$

with a $B>0$. Then the solution $(x(s), y(s))$ circles clockwise around the origin. Let $s_{i}>s_{0}$ and $p_{i}>0$ with

$$
\left(x\left(s_{i}\right), y\left(s_{i}\right)\right)=\left(0, p_{i}\right) \quad \text { for } \quad i=1,2 .
$$

Define a Liapunov function

$$
V(x, y)=\frac{1}{2} y^{2}+G(x)
$$


so that

$$
\dot{V}_{(3.1)}(x, y)=x g(x)>0 \quad \text { if } \quad x \neq 0 .
$$

If $s_{1}<s_{2}$, then $p_{1}<p_{2}$. In fact, we have

$$
\frac{1}{2} p_{1}^{2}=V\left(x\left(s_{1}\right), y\left(s_{1}\right)\right)<V\left(x\left(s_{2}\right), y\left(s_{2}\right)\right)=\frac{1}{2} p_{2}^{2} .
$$

Hence, there exists a simple closed curve $C$ surrounding the origin such that

$$
\operatorname{dist}\{(x(s), y(s)), C\} \rightarrow 0 \quad \text { as } \quad s \rightarrow \infty .
$$

Let $\varepsilon_{0}>0$ be sufficiently small and define

$$
l=\max \left\{g(x): \varepsilon_{0} \leq x \leq B\right\}, \quad m=\min \left\{x g(x): \varepsilon_{0} \leq x \leq B\right\} .
$$

By (4.2) the solution $(x(s), y(s))$ does not stay in $\left\{(x, y):|x|<\varepsilon_{0}\right\}$. Hence, there exist sequences $\left\{\tau_{n}\right\}$ and $\left\{\sigma_{n}\right\}$ with $s_{0}<\tau_{n}<\sigma_{n}<\tau_{n+1}$ and $\tau_{n} \rightarrow \infty$ as $n \rightarrow$ $\infty$ such that

$$
x\left(\tau_{n}\right)=x\left(\sigma_{n}\right)=\varepsilon_{0}, \quad y\left(\tau_{n}\right)>\varepsilon_{0}, \quad y\left(\sigma_{n}\right)<-\varepsilon_{0}
$$

and

$$
x(s)>\varepsilon_{0} \quad \text { for } \quad \tau_{n}<s<\sigma_{n} .
$$

We have

$$
-2 \varepsilon_{0}>y\left(\sigma_{n}\right)-y\left(\tau_{n}\right)=-\int_{\tau_{n}}^{\sigma_{n}} g(x(s)) d s \geq-l\left(\sigma_{n}-\tau_{n}\right),
$$

and therefore, for $s>\sigma_{n}$

$$
\begin{aligned}
V(x(s), y(s))-V\left(x\left(s_{0}\right), y\left(s_{0}\right)\right) & =\int_{s_{0}}^{s} x(\sigma) g(x(\sigma)) d \sigma \geq \sum_{k=1}^{n} \int_{\tau_{k}}^{\sigma_{k}} x(s) g(x(s)) d s \\
& \geq m \sum_{k=1}^{n}\left(\sigma_{k}-\tau_{k}\right) \geq \frac{2 m \varepsilon_{0}}{l} n
\end{aligned}
$$

which tends to $\infty$ as $n \rightarrow \infty$. This is a contradiction to (4.1) and completes the proof.

Let

$$
V(x, y)=\frac{1}{2} y^{2}+G(x)
$$

and consider the curve

$$
V(x, y)=V\left(x_{0}, y_{0}\right)
$$

where $x_{0}>0$. Then there exist two points of intersection of the curve with the line $y=-x$. In fact, the equation

$$
V(x,-x)=V\left(x_{0}, y_{0}\right)
$$

has exactly two roots because $V(x,-x)$ is increasing for $x>0$ and decreasing for $x<0$, and $V(0,0)=0$. Let $(-a, a)$ and $(b,-b)$ be the intersection points, where $a>0$ and $b>0$. Define

$$
S=\left\{(x, y):-a \leq x \leq c \text { and } V(x, y) \leq V\left(x_{0}, y_{0}\right)\right\}
$$


in which $c=\max \left\{b, x_{0}\right\}$. Then it is clear that $S$ is a bounded set. Lemma 4.1 shows that every solution of (3.1) starting in $S \backslash\{0\}$ does not remain in $S$. Take note of the vector field of (3.1) and the fact that

$$
\dot{V}_{(3.1)}(x, y)=x g(x)>0 \quad \text { if } \quad x \neq 0 .
$$

Then we also see that every solution of (3.1) starting in $S^{c}$, the complement of $S$ in $\mathbf{R}^{2}$, stays in $S^{c}$ for all future time. Thus, we have

Lemma 4.2. Every solution of (3.1) starting in $S \backslash\{0\}$ enters $S^{c}$ which is a positive invariant set with respect to (3.1).

Proof of Theorem 1.2. We prove only the case that condition (1.6) is satisfied for $x>R$, because the other case is carried out in the same way.

First, we will show that system (3.1) fails to have property $\left(X^{+}\right)$in the right half-plane. If $G(\infty)<\infty$, then this fact is clear because of Lemma 3.3. Suppose that $G(\infty)=\infty$. To use Lemma 3.4, we will check that conditions (3.2)-(3.4) hold.

Let

$$
h(r)=\frac{r}{4(\log r)^{2}}
$$

for $r$ sufficiently large. Then $\frac{h(r)}{r}$ is nonincreasing and nonnegative; and we have

$$
r\left(\int_{r}^{\infty} \frac{h(\xi)}{\xi^{2}} d \xi\right)^{2}=\frac{r}{16(\log r)^{2}}=\frac{1}{4} h(r),
$$

that is, conditions (3.3) and (3.4) are satisfied. Define continuous functions $k(x)$ and $L(x)$ on $\mathbf{R}$ by

$$
k(x)=\left(\frac{\lambda}{\log x}\right)^{2} x \quad \text { and } \quad L(x)=\left(\frac{\nu x}{\log x}\right)^{2}
$$

for $x>R$ with $\lambda^{2}<2 \nu^{2}<\frac{1}{16}$. Then

$$
K(x) \equiv \int_{0}^{x} k(\xi) d \xi
$$

is increasing for $x>R$, and there exist constants $M>0$ and $N>0$ such that

$$
L(x)+M \geq K(x)
$$

and

$$
G(x) \leq \frac{1}{8} x^{2}+K(x)+N
$$

for $x>0$. Hence, we obtain

$$
\begin{aligned}
-\frac{1}{2} u h(u)+\frac{1}{8}(h(u))^{2}+K(2 u-h(u)) & \leq-\frac{u^{2}}{8(\log u)^{2}}+\frac{u^{2}}{128(\log u)^{4}}+K(2 u) \\
& \leq-\frac{u^{2}}{8(\log u)^{2}}+\frac{u^{2}}{128(\log u)^{4}}+L(2 u)+M \\
& \leq-\frac{\left(1-32 \nu^{2}\right) u^{2}}{8(\log u)^{2}}+\frac{u^{2}}{128(\log u)^{4}}+M \\
& \rightarrow-\infty \text { as } u \rightarrow \infty
\end{aligned}
$$


and therefore, for $u$ sufficiently large

$$
\begin{aligned}
\frac{1}{2} u^{2} & \geq \frac{1}{2} u^{2}-\frac{1}{2} u h(u)+\frac{1}{8}(h(u))^{2}+K(2 u-h(u))+N \\
& =\frac{1}{8}(2 u-h(u))^{2}+K(2 u-h(u))+N \\
& \geq G(2 u-h(u)) .
\end{aligned}
$$

Let $u=\sqrt{2 G(x)}$. Then we have

$$
x \geq 2 \sqrt{2 G(x)}-h(\sqrt{2 G(x)})
$$

for $x$ sufficiently large. Thus, condition (3.2) is also satisfied, and so system (3.1) fails to have property $\left(X^{+}\right)$in the right half-plane by Lemma 3.3. Hence, there exists a point $P_{0}\left(x_{0}, y_{0}\right)$ with $x_{0} \geq 0$ and $y_{0}>-x_{0}$ such that $\gamma^{+}\left(P_{0}\right)$ runs to infinity without intersecting the curve $y=-x$.

We here suppose that (1.1) has an oscillatory solution. Let $\gamma^{+}(Q)$ be the positive semitrajectory which corresponds to the oscillatory solution of (1.1). By virtue of Lemma 4.2, we see that $\gamma^{+}(Q)$ eventually goes around the set $S$ infinitely many times. Hence, it crosses the half-line $\left\{(x, y): x=x_{0}\right.$ and $\left.y>y_{0}\right\}$ at a point $P_{1}\left(x_{0}, y_{1}\right)$ with $y_{1}>y_{0}$. From the uniqueness of solutions for the initial value problem, it turns out that

(i) $\gamma^{+}(Q)$ coincides with $\gamma^{+}\left(P_{1}\right)$ except for the arc $Q P_{1}$.

(ii) $\gamma^{+}\left(P_{1}\right)$ lies above $\gamma^{+}\left(P_{0}\right)$.

Hence, $\gamma^{+}(Q)$ runs to infinity without crossing the curve $y=-x$. This contradicts the fact that $\gamma^{+}(Q)$ circles the set $S$. The proof is now complete.

\section{REFERENCES}

[1] T. Hara and J. Sugie, When all trajectories in the Liénard plane cross the vertical isocline?, Nonlin. Diff. Eq. Appl. 2 (1995), 527-551.

[2] T. Hara, T. Yoneyama and J. Sugie, Continuation results for differential equations by two Liapunov functions, Ann. Mat. Pura Appl. 133 (1983), 79-92. MR 85k:34009

[3] E. Hille, Non-oscillation theorems, Tran. Amer. Math. Soc. 64 (1948), 234-252. MR 10:376

[4] J. Sugie, Continuation results for differential equations without uniqueness by two Liapunov functions, Proc. Japan Acad. Math. Sci. Ser. A 60 (1984), 153-156. MR 86a:34006

[5] _ Global existence and boundedness of solutions of differential equations, doctoral dissertation, Tôhoku University, 1990.

[6] C. A. Swanson, Comparison and oscillation theory of linear differential equations, Academic Press, New York and London, 1968. MR 57:3515

Department of Mathematics, Faculty of Science, Shinshu University, Matsumoto 390 , JAPAN

Current address: Department of Mathematics and Computer Science, Shimane University, Matsue 690, Japan

E-mail address: jsugie@botan.shimane-u.ac.jp

Department of Mathematical Sciences, University of Osaka Prefecture, Sakai 593, JAPAN

E-mail address: hara@ms.osakafu-u.ac.jp 